\title{
Evaluation of sepsis induced cardiac dysfunction as a predictor of mortality
}

\author{
Amarja Ashok Havaldar(D
}

\begin{abstract}
Background: Sepsis is characterized by life threatening organ dysfunction with dysregulated immune response. Cardiac dysfunction seen in sepsis is unique as it is reversible within 7-10 days. Initial study by Parker et al. in 1984, showed, paradoxically lower ejection fraction in survivors of septic shock. Subsequent meta-analysis did not support that survivors had lower ejection fraction. Aim of our study was to assess the sepsis induced cardiac dysfunction by 2D echocardiography and Troponin I.

Methods: After obtaining institutional ethical committee approval (ref 125/2016), a prospective observational study was done in an university medical college from February 2016 to April 2016. Inclusion criteria were patients diagnosed with sepsis by new sepsis definition. Pregnant patients and patients with poor echo window were excluded. Echocardiographic assessment was done within $48 \mathrm{~h}$ of diagnosis of sepsis by standard methods. Primary outcome was ICU mortality and secondary outcome was ICU length of stay. Statistical analysis was done using STATA ${ }^{\text {TM }}$ (Version14, College station TX).

Results: Fifty eight patients were screened, ten were excluded due to poor echo window. Baseline characteristics were similar in survivors and non survivors, except APACHE II, SOFA age and cumulative fluid balance. Echocardiographic parameters, mitral annular plane systolic excursion (MAPSE), E/e' and LV systolic function assessed by visual gestalt method were found to be statistically significant. Parameters found significant in bivariate analysis were used as a covariate in logistic regression. APACHE II and MAPSE were significant co-variates in logistic regression with ROC (0.95) and calibration was satisfactory (chi2(df8), 1.98, $p=0.98)$.
\end{abstract}

Conclusions: Sepsis induced cardiac dysfunction assessed by echocardiography showed measurement of MAPSE when combined with APACHE II was a good predictor of mortality. Among the echocardiographic parameters MAPSE alone was a good predictor of mortality. Results of this study need further validation from larger study.

Keywords: Sepsis, Septic cardiomyopathy, Troponin I, Vasoplegia

\section{Background}

Sepsis is characterized by dysregulated immune response with life threatening organ dysfunction [1-3]. This is one of the major cause of mortality in intensive care unit. Septic shock is characterized by low systemic vascular resistance and it has two phases of shock. Early hyperdynamic phase of septic shock has high cardiac output and late hypodynamic phase is characterized by reduced cardiac output [4-8]. Cardiac dysfunction seen in patients with sepsis can be of different types, systolic, diastolic or both and etiology of sepsis may affect the type of cardiac dysfunction. To explain it further, sepsis due to underlying lung pathology can result in elevation of right

Correspondence: amarjahavaldar@rediffmail.com

MICU, St John's Medical College, Kormangala, Bangalore 560034, India ventricular (RV) afterload. High RV afterload leads to less pulmonary blood flow and reduces the possibility of left ventricular (LV) diastolic failure if LV function is normal prior to sepsis [4, 9-12].

Sepsis induced cardiac dysfunction was first described by Parker et al., in the study of 20 patients. These patients had hematological and solid organ malignancies and survivors had lower ejection fraction which was considered as an adaptive response to overcome vasoplegia [4-6].

However, recent meta-analysis on sepsis induced ventricular dysfunction showed no difference in ejection fraction (EF) between survivors and non survivors [13].

Cardiac specific biomarker such as Troponin I has been studied in septic patients and elevated Troponin I was associated with higher risk of mortality $[14,15]$. The aim

(c) The Author(s). 2018 Open Access This article is distributed under the terms of the Creative Commons Attribution 4.0 International License (http://creativecommons.org/licenses/by/4.0/), which permits unrestricted use, distribution, and 
of the current study was to assess prognostic significance of sepsis induced cardiac dysfunction evaluated by echocardiography and Troponin I.

\section{Methods}

Formal consent from institutional ethical committee was obtained prior to initiating the study (ref no125/2016). It was a prospective observational study conducted for a duration of 3 months from February 2016 to April 2016 in the 30 bedded ICU of a large university medical center. Patients were included, after obtaining consent from patients or legally acceptable representatives. All patients above 18 years of age with the diagnosis of sepsis were included. Patients with poor echo window and pregnant patients were excluded from the study. A convenience sampling method was used for patient selection.

Baseline characteristics, APACHE II and SOFA scores were calculated within $24 \mathrm{~h}$ of ICU admission. APACHE II score takes into account, clinical, laboratory, age and comorbidities. It indicates severity of illness. Similarly, SOFA score considers number of organ failures. Echocardiographic parameters and Troponin I were collected within $48 \mathrm{~h}$ of diagnosis of sepsis. All patients were followed up till the ICU discharge and the primary outcome of interest was ICU mortality.

Echocardiographic measurements were done using a Sonosite $^{\text {Th }}$ Edge portable machine with a $2.5 \mathrm{MHz}$ probe and inbuilt cardiac calculation software. LV systolic function was assessed by visual gestalt method, Simpson's method, fractional shortening and MAPSE. By visual gestalt method, cardiac function was classified into hyperdynamic, normal, mild, moderate, and severe LV dysfunction. By Simpson's method readings were taken in apical 4 chamber view and EF was calculated. LV fractional shortening was assessed by M-mode echocardiography. By using $\mathrm{M}$ - mode at lateral mitral annulus MAPSE was measured. LV diastolic dysfunction was assessed by transmitral flow velocity (E, A and deceleration time DCT) were measured. E/A was calculated. Assessment of mitral annular flow velocity was done using tissue doppler imaging. Lateral mitral annular flow velocities, e' and a' were measured. E/A, E/e' were calculated. In patients having regional wall motion abnormalities (RWMA), both lateral and septal mitral leaflet annular velocities were measured and averaged. The right ventricular systolic function was assessed using tricuspid annular plane systolic excursion (TAPSE). Tei index was calculated which is a measure of myocardial performance. Ventriculoarterial elastance was calculated by taking the ratio of end systolic volume (ESV) by stroke volume (SV). All measurements were performed by the first author to avoid inter-observer bias. An average of consecutive three recordings was taken to avoid intra-observer bias.

Statistical analysis was done using STATA ${ }^{\text {тм }}$ (Version14, College station TX). Data was expressed as mean (SD) or median (Interquartile range IQR) as appropriate. Continuous variables were analysed by ' $t$ ' test (Satterwhaite's for unequal variance) and categorical variables were analysed using ' $X$ ', test. 'Mann-Whitney $U$ ' test was used for nonparametric continuous variables. A standard 95\% confidence limit and a $p$ value at 0.05 were used for assessing statistical significance. Variables identified as significant in the bivariate analysis were used as independent variables and outcome was used as dependent variable to develop a predictive model using logistic regression.

\section{Results}

Fifty eight patients were screened. Ten patients (17.24\%) were excluded due to poor echo window. Out of fourty eight patients, 29 patients (60.41\%) survived and 19 patients (39.58\%) died. Among the baseline characteristics, age (65.94 vs 54.89), APACHE II (25.84 vs 16.34), SOFA (9 vs 4) and higher cumulative fluid balance of first 3 days were statistically significant. Preexisting comorbidities did not had any effect on the outcome (Table 1).

Cardiac biomarker Troponin I was not statistically significant $(0.08$ vs 0.03$)$ in both groups $(p=0.051)$ (Table 1). Echocardiographic parameters were similar among survivors and non survivors except for the MAPSE, E/e' and LV systolic dysfunction assessed by visual gestalt method. Assessment of LV systolic function by this method showed hyperdynamic LV (12.2\%), normal (26.83\%), mild (19.51\%), moderate (26.83\%) and severe LV dysfunction in (14.63\%) patients (Table 2). Severe LV systolic dysfunction was seen in nonsurvivors as compared to survivors.

Multivariable logistic regression analysis was done using echocardiographic parameters which were found statistically significant in bivariate analysis. It showed MAPSE was the only significant parameter (Table 3).

Table 1 Baseline characteristics

\begin{tabular}{llll}
\hline Baseline Parameters & Survivors $n=29$ & Non Survivors $n=19$ & $P$ value \\
\hline Age & $54.89(12.61)$ & $65.94(11.51)$ & $0.003^{*}$ \\
APACHE II & $16.34(4.91)$ & $25.84(6.44)$ & $<0.001^{*}$ \\
SOFA & $4(3-6)$ & $9(8-12)$ & $<0.001^{*}$ \\
Fluid balance & $975(-182-2495)$ & $3654(3026.7-5848)$ & $<0.001^{*}$ \\
Lactate & $2.10(1.2-4.4)$ & $6.3(2.10-7.20)$ & 0.06 \\
Troponin Day 1 & $0.03(0.01-0.11)$ & $0.08(0.04-1.68)$ & 0.0503 \\
Comorbidities & & & \\
Diabetes Mellitus & $10(34.48)$ & $9(47.36)$ & 0.37 \\
IHD & $4(13.33)$ & $5(27.77)$ & 0.27 \\
Hypertension & $9(31.03)$ & $8(42.10)$ & 0.43 \\
CKD & $2(6.89)$ & $2(10.52)$ & 0.65 \\
CLD & $3(10.34)$ & $0(0)$ & 0.14 \\
COPD & $1(3.44)$ & $0(0)$ & 0.41 \\
\hline *
\end{tabular}

significant $p<0.05$ 
Table 2 Echocardiographic parameters

\begin{tabular}{|c|c|c|c|}
\hline ECHO parameters & Survivors (29) & NonSurvivors (19) & $p$ value \\
\hline EF M mode & $58.73(9.85)$ & $59.08(19.48)$ & 0.52 \\
\hline EF Simpson's Method & $30.85(14.26)$ & $36.31(13.98)$ & 0.89 \\
\hline MAPSE & $1.54(1.39-1.69)$ & $1.12(0.90-1.35)$ & $0.026^{*}$ \\
\hline LV systolic function Visual Gestalt & & & $0.02^{*}$ Fischer's Exact \\
\hline Hyperdynamic & $3 / 5$ & $2 / 5$ & \\
\hline Normal & $10 / 11$ & $1 / 11$ & \\
\hline Mild & $7 / 8$ & $1 / 8$ & \\
\hline Moderate & $7 / 11$ & $4 / 11$ & \\
\hline Severe & $1 / 6$ & $5 / 6$ & \\
\hline E/A & $1.04(0.40)$ & $1.30(0.59)$ & 0.95 \\
\hline $\mathrm{DCT}(\mathrm{ms})$ & $122.63(43.35)$ & $128.22(55.30)$ & 0.71 \\
\hline $\mathrm{S}(\mathrm{cm} / \mathrm{sec})$ & $9.83(2.98)$ & 10(3.58) & 0.87 \\
\hline$e^{\prime}(\mathrm{cm} / \mathrm{sec})$ & $11.22(2.70)$ & $10.27(3.21)$ & 0.30 \\
\hline$E / e^{\prime}$ & $6.32(1.75)$ & $8.03(2.79)$ & $0.025^{*}$ \\
\hline ESV (ml) & $47.1(36-79.8)$ & $45.95(27.45-73.80)$ & 0.72 \\
\hline $\mathrm{SV}(\mathrm{ml})$ & $27(14-37.1)$ & $23.9(13.7-32.15)$ & 0.65 \\
\hline $\mathrm{EDV}(\mathrm{ml})$ & 69(58.4-104.6) & 70.65(56.2-115.70) & 0.83 \\
\hline VTI (cm/sec) & 15.14(3.13) & 13.93(3.19) & 0.19 \\
\hline VA elastance & $2.42(1.46-3.78)$ & 1.68(1.38-3.18) & 0.67 \\
\hline Tei Index & $0.61(0.19)$ & $0.64(0.27)$ & 0.635 \\
\hline
\end{tabular}

"significant $p<0.05$

The receiver operating characteristics (ROC) for parameter MAPSE showed good discrimination (Area under the curve [AUC 0.822]) as against poor discrimination for E/e' (AUC 0.67) and fair discrimination for LV systolic function (AUC 0.71) (Figs. 1, 2 and 3).

In developing a predictive model using echocardiographic and clinically important parameters we found only APACHE II and MAPSE were found to be significant co-variates. (APACHE II coef $0.4869 p=0.02$, MAPSE $-9.3672 p=0.04$, constant 0.7109 ). The discrimination of this model was excellent (receiver operating characteristics [ROC 0.95]) and calibration was satisfactory (chi2(df8),1.98, $p=0.98$ ) (Fig. 4) as against each parameter when taken individually. (ROC for APACHE was AUC 0.88 Fig. 5).

Among the echocardiographic parameters, MAPSE alone was found to be the predictor of mortality with coef $-3.7, p=0.03$ and constant 1.21 .

\section{Discussion}

Sepsis induced cardiac dysfunction was evaluated in fourty eight patients. Echocardiographic parameters such as MAPSE, E/e' and LV systolic dysfunction by visual gestalt were the significant parameters. In multivariate analysis MAPSE and APACHE II were significant covariates. In our study MAPSE when combined with APACHE II could predict the outcome in patients of sepsis.

Initial small study of 20 patients evaluating sepsis induced cardiac dysfunction showed patients with lower ejection fraction had better survival. Patients included in the study were predominantly elderly and with hematological or solid organ malignancy. Repeat echocardiographic assessment after day 7 showed improved ejection fraction in survivors, thereby suggesting reversibility of sepsis induced cardiac dysfunction [6]. Subsequent meta-analysis done showed no difference in ejection fraction among survivors and

Table 3 Multivariable logistic regression of echo parameters

\begin{tabular}{lllllll}
\hline Mortality & Coefficient $(\beta)$ & SE & $z$ & Odds Ratio & $p$ value \\
\hline MAPSE & -3.7 & 1.71 & -2.17 & 0.024 & 0.030 & $0.00-0.69$ \\
E/e' & 0.31 & 0.25 & 1.23 & 1.37 & 0.219 & $0.82-2.29$ \\
LV systolic & 0.44 & 0.40 & 1.09 & 1.56 & 0.275 & $0.70-3.47$ \\
Visual Gestalt & & & & 3.37 & 6.72 \\
Constant & 1.21 & 2.8 & & &
\end{tabular}

significant $p<0.05$ 


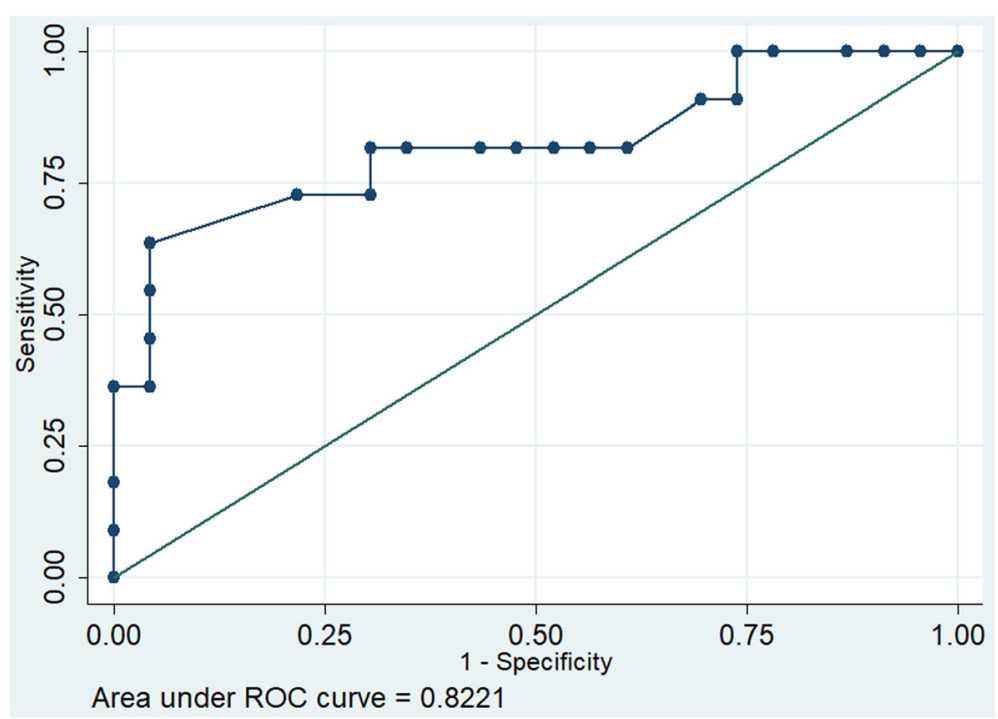

Fig. 1 Receiver operating characteristics of MAPSE

non survivors. It also showed non indexed left ventricular parameters were mildly increased in survivors. The indexed parameters were obtained by dividing the echocardiographic parameter by body surface area (BSA). The results of the metaanalysis were not easily generalizable due to wide heterogeneity [13].

In earlier studies cardiac function was assessed by invasive methods [6]. Echocardiography has helped to evaluate cardiac function noninvasively and at repeated intervals during each stage of disease process to decide timely intervention [16-19]. In our study, echocardiographic assessment of LV systolic function was done by different methods, which showed MAPSE as the significant covariate in logistic regression. MAPSE is easy to measure and interpret and is indicative of assessment of longitudinal systolic function. In patients with impaired systolic function had lower values of MAPSE [20-22]. Previous studies have found good correlation between MAPSE and left ventricular longitudinal strain [20-22].

In prospective cohort of 50 patients, MAPSE and SOFA score was found to be a good predictive model [20]. Similar findings were seen in the current study, and MAPSE along with APACHE II score was a good predictive model in patients with sepsis.

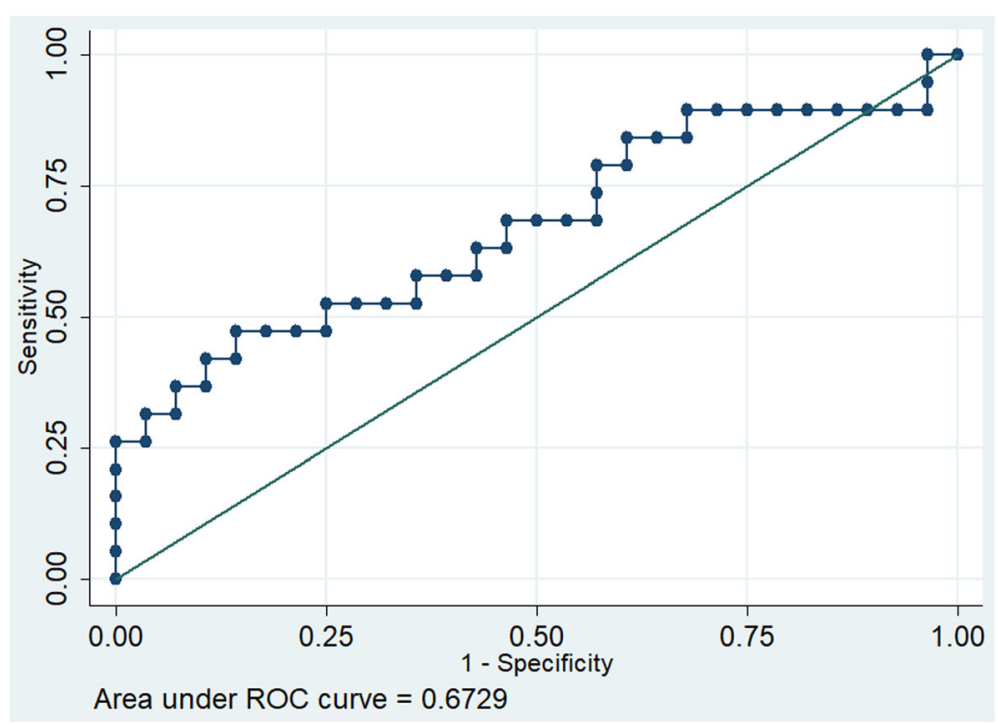

Fig. 2 Receiver operating characteristics of E/e 


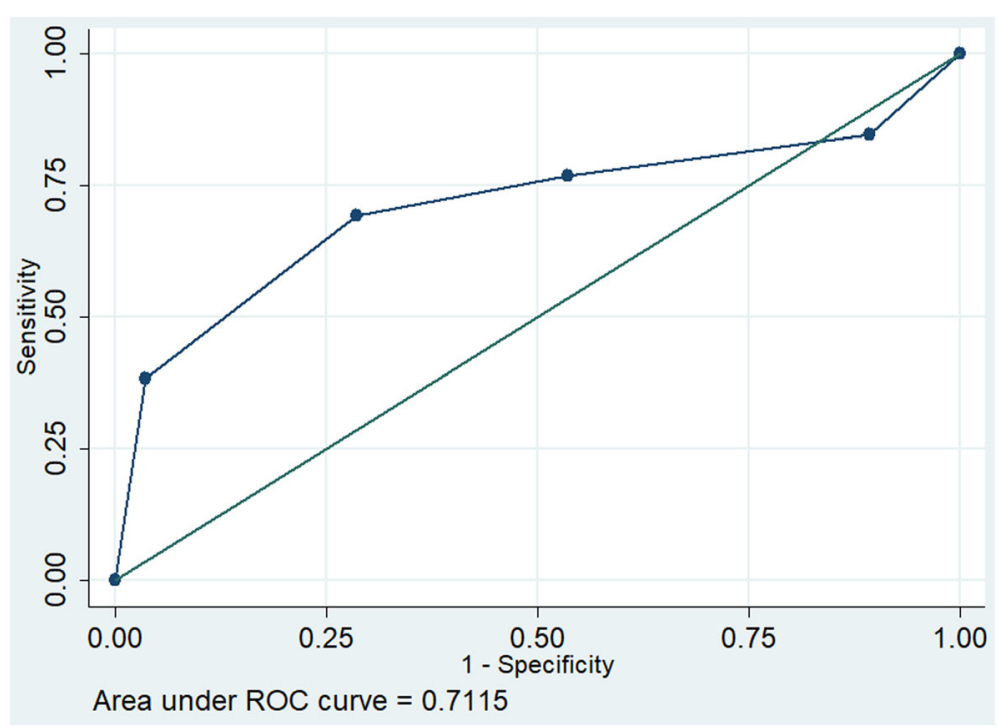

Fig. 3 Receiver operating characteristics of LV systolic function

Cardiac biomarker such as Troponin I elevation in sepsis is due to inflammatory and endothelial factors. It is also influenced by vasopressor support and concomitant renal injury [13]. Elevation of cardiac biomarker, Troponin I in sepsis patients had higher risk of death (OR 1.92) as shown by the meta-analysis [14]. In our study Troponin I was not found to be a significant parameter.

There were certain limitations to our study. This was single centre study. All observations were collected by single observer. To minimize intraobserver variability, average of the three readings were obtained. The assessment of cardiac function was done within $48 \mathrm{~h}$ of admission. The patients developed cardiac dysfunction after $48 \mathrm{~h}$ were not included in the study. Effect of duration of sepsis and changes in echocardiographic parameters could not be tested. There was only one echocardiographic assessment, so reversibility of cardiac dysfunction could not be evaluated. Echocardiographic assessment was also influenced by use of vasopressors or inotropic agents. Systolic function assessment by MAPSE evaluates

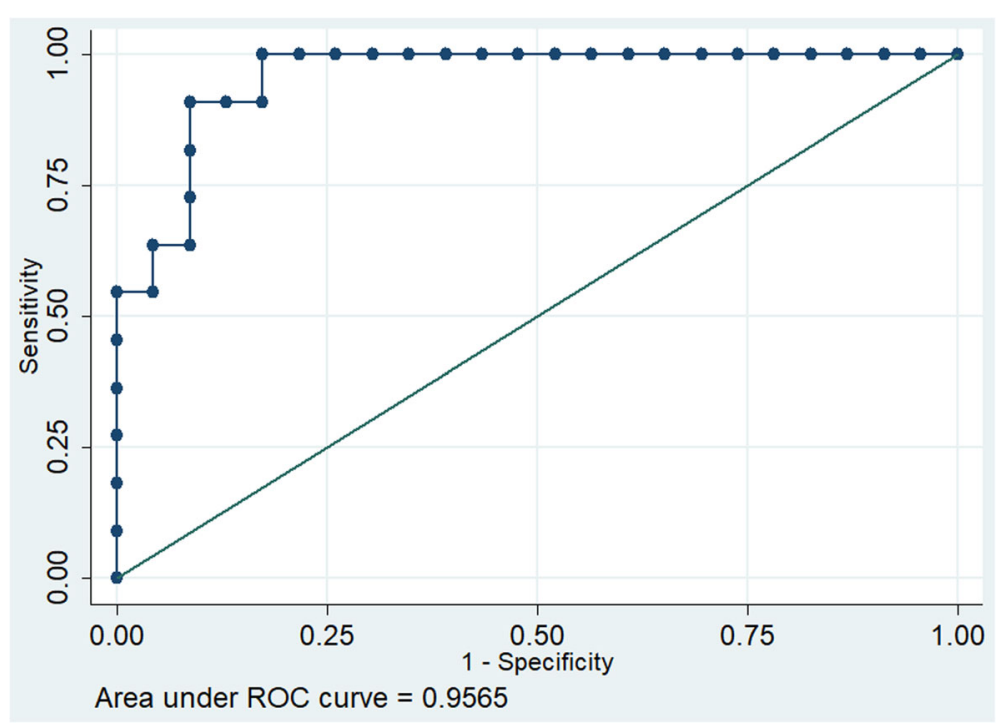

Fig. 4 Receiver operating characteristics of MAPSE and APACHE I| 


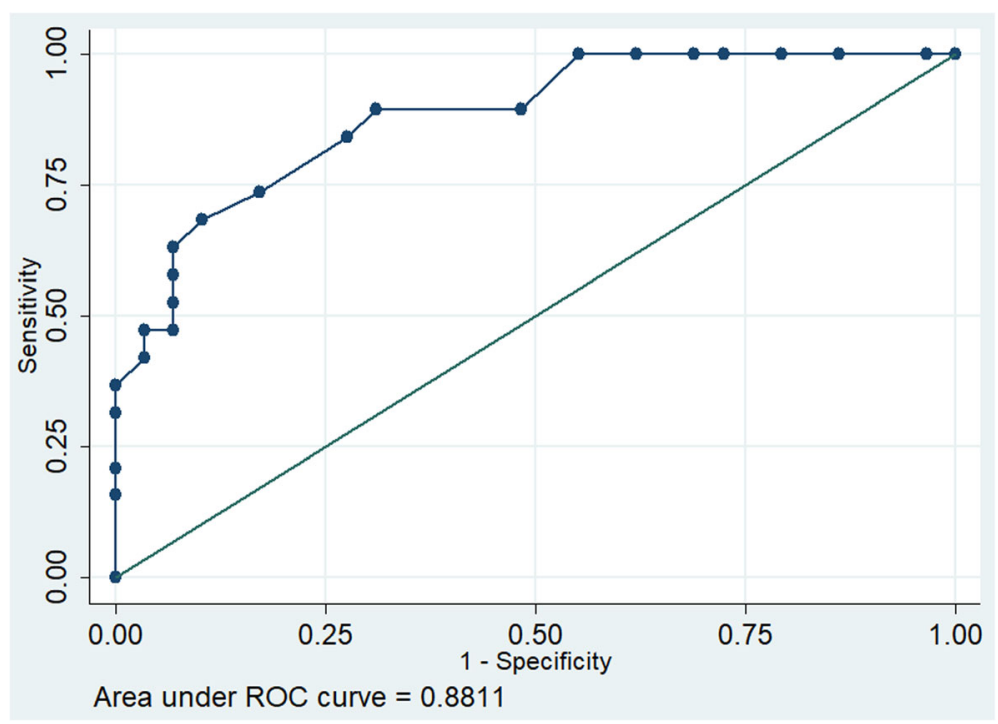

Fig. 5 Receiver operating characteristics of APACHE ॥

mainly longitudinal LV function. It's role in predicting global LV function needs to be evaluated in further studies. Measurement of continuous cardiac output would have helped in studying the evolution of shock and effect of different therapeutic interventions over a period of time.

\section{Conclusion}

This small study has shown that MAPSE when combined with APACHE II score is a good predictor of mortality. Among the echocardiographic parameters MAPSE alone was a good predictor of mortality. Cardiac biomarker Troponin I was not the significant parameter in our study. Assessment of cardiac function in sepsis patient is important and serial evaluation and measuring of the MAPSE may help to monitor the progress of the patient. Future studies involving more number of patients and multiple observers is indicated to validate results of this study.

\section{Abbreviations}

APACHE: Acute physiology, age and chronic health evaluation; AUC: Area under the curve; DCT: Deceleration time; EF: Ejection fraction; ESV: End systolic volume; ICU: Intensive care unit; IQR: Interquartile range; LV: Left ventricle; MAPSE: Mitral annular plane systolic excursion; ROC: Receiver operating characteristics; RV: Right ventricle; RWMA: Regional wall motion abnormalities; SD: Standard deviation; SOFA: Sequential organ failure assessment; SV: Stroke volume; TAPSE: Tricuspid annular plane systolic excursion; VTI: Velocity time integral

\section{Acknowledgements}

I personally thank all the ICU staff who helped in data collection.

\section{Funding}

Not applicable.

\section{Availability of data and materials}

The datasets used and analysed in the present study are available from the corresponding author upon reasonable request.

\section{Author's contribution}

AAH had helped in initiation, data collection and drafted the manuscript.

The author read and approved the final manuscript.

\section{Ethics approval and consent to participate}

Ethics committee of St Johns Medical college, Bangalore has approved the study. (ref 125/2016).

\section{Consent for publication}

Not applicable.

\section{Competing interests}

The author declares that she has no competing interests.

\section{Publisher's Note}

Springer Nature remains neutral with regard to jurisdictional claims in published maps and institutional affiliations.

Received: 18 September 2018 Accepted: 20 November 2018 Published online: 30 November 2018

\section{References}

1. Singer M, Deutschman CS, Seymour CW, Shankar-Hari M, Annane D, Bauer $\mathrm{M}$, et al. The third international consensus definitions for sepsis and septic shock (sepsis-3). JAMA. 2016;315(8):801-10.

2. Abraham E. New definitions for sepsis and septic shock: continuing evolution but with much still to be done. JAMA. 2016;315(8):757-9.

3. Seymour CW, Liu VX, Iwashyna TJ, Brunkhorst FM, Rea TD, Scherag A, et al. Assessment of clinical criteria for sepsis: for the third international consensus definitions for Sepsis and septic shock (Sepsis-3). JAMA. 2016; 315(8):762-74

4. J Romero-Bermejo F, Ruiz-Bailen M, Gil-Cebrian J, J Huertos-Ranchal M. Sepsis-induced cardiomyopathy. Curr Cardiol Rev. 2011;7(3):163-83.

5. Vieillard-Baron A. Septic cardiomyopathy. Ann Intensive Care. 2011;1(1):6. 
6. Parker MM, Shelhamer JH, Bacharach SL, Green MV, Charles N, Frederick TM, et al. Profound but reversible myocardial depression in patients with septic shock. Ann Intern Med. 1984;100(4):483-90.

7. Flynn A, Mani BC, Mather PJ. Sepsis-induced cardiomyopathy: a review of pathophysiologic mechanisms. Heart Fail Rev. 2010;15(6):605-11.

8. Werdan K, Oelke A, Hettwer S, Nuding S, Bubel S, Hoke R, et al. Septic cardiomyopathy: hemodynamic quantification, occurrence, and prognostic implications. Clin Res Cardiol. 2011;100(8):661-8.

9. Rolando G, Espinoza ED, Avid E, Welsh S, Pozo JD, Vazquez AR, et al. Prognostic value of ventricular diastolic dysfunction in patients with severe sepsis and septic shock. Rev Bras Ter Intensive. 2015;27(4):333-9.

10. Celes MR, Prado CM, Rossi MA. Sepsis: going to the heart of the matter. Pathobiology. 2013;80(2):70-86.

11. Furian T, Aguiar C, Prado K, Ribeiro RV, Becker L, Martinelli N, et al. Ventricular dysfunction and dilation in severe sepsis and septic shock: relation to endothelial function and mortality. J Crit Care. 2012;27(3):319-e9.

12. Kakihana Y, Ito T, Nakahara M, Yamaguchi K, Yasuda T. Sepsis-induced myocardial dysfunction: pathophysiology and management. J Intensive Care. 2016:4(1):22.

13. Huang SJ, Nalos M, McLean AS. Is early ventricular dysfunction or dilatation associated with lower mortality rate in adult severe sepsis and septic shock? A meta-analysis. Crit Care. 2013:17(3):R96.

14. Bessière F, Khenifer S, Dubourg J, Durieu I, Lega JC. Prognostic value of troponins in sepsis: a meta-analysis. Intensive Care Med. 2013;39(7):1181-9.

15. Reynolds T, Cecconi M, Collinson P, Rhodes A, Grounds RM, Hamilton MA. Raised serum cardiac troponin I concentrations predict hospital mortality in intensive care unit patients. Br J Anaesth. 2012;109(2):219-24.

16. McLean AS. Echocardiography in shock management. Crit Care. 2016;20(1):275.

17. Repessé X, Charron C, Vieillard-Baron A. Evaluation of left ventricular systolic function revisited in septic shock. Crit Care. 2013;17(4):164.

18. De Geer L, Engvall J, Oscarsson A. Strain echocardiography in septic shock-a comparison with systolic and diastolic function parameters, cardiac biomarkers and outcome. Crit Care. 2015;19(1):122.

19. Via G, Price S, Storti E. Echocardiography in the sepsis syndromes. Crit Ultrasound J. 2011:3(2):71.

20. Bergenzaun L, Öhlin H, Gudmundsson P, Willenheimer R, Chew MS. Mitral annular plane systolic excursion (MAPSE) in shock: a valuable echocardiographic parameter in intensive care patients. Cardiovasc Ultrasound. 2013:11(1):16.

21. Hu K, Liu D, Herrmann S, Niemann M, Gaudron PD, Voelker W, Ertl G, Bijnens B, Weidemann F. Clinical implication of mitral annular plane systolic excursion for patients with cardiovascular disease. Eur Heart J-Cardiovasc Imaging. 2012;14(3):205-12.

22. Huang SJ, Ting I, Huang AM, Slama M, McLean AS. Longitudinal wall fractional shortening: an M-mode index based on mitral annular plane systolic excursion (MAPSE) that correlates and predicts left ventricular longitudinal strain (LVLS) in intensive care patients. Crit Care. 2017;21(1):292.

Ready to submit your research? Choose BMC and benefit from:

- fast, convenient online submission

- thorough peer review by experienced researchers in your field

- rapid publication on acceptance

- support for research data, including large and complex data types

- gold Open Access which fosters wider collaboration and increased citations

- maximum visibility for your research: over $100 \mathrm{M}$ website views per year

At $\mathrm{BMC}$, research is always in progress.

Learn more biomedcentral.com/submissions 